\title{
Recherche comparée sur la prise de parole de certains élèves du préscolaire au primaire en français langue maternelle en France et au Québec
}

\author{
Lizanne Lafontaine (Université du Québec en Outaouais) \\ Catherine Le Cunff. (Université Paris X France)
}

Résumé: Cette recherche franco-québécoise vise à valider ou à corriger des situations et des démarches isolées en enseignement de l'oral qui n'ont pu faire l'objet d'une expérimentation suffisamment large. Nous poursuivons l'objectif général suivant : mieux connaître les représentations de certains enseignants québécois et français concernant l'oral et sa place dans les apprentissages comme dans la socialisation au sein de la classe quand ils mettent en place des situations innovantes du préscolaire au secondaire. Nous expliquons les différences méthodologiques entre les deux pays qui nous ont quand même permis d'établir des convergences ainsi que des divergences importantes entre les divers ordres d'enseignement. Nous analysons également des extraits de corpus québécois et français qui démontrent que l'oral doit être enseigné de façon systématique à l'école au Québec et en France et qu'un continuum plus présent doive se faire sentir dans les programmes d'études entre la fin du primaire et le début du secondaire.

Abstract: This French-Quebecois research aims to validate or to correct isolated situations or processes in teaching of oral skills that can't be conducted in a large scale experiment. We seek the following general objectives: to better understand some French and Quebecois teachers' representations regarding teaching oral skills and its place in learning as well as in socialization in the classroom when these teachers present innovative situations from preschool to the secondary. We explain the methodological differences from the two countries although they allow us to establish the important convergences and divergences between the various education levels. We also analyse excerpts of the Quebecois and French corpus to demonstrate that oral skills need to be taught systematically in Quebec and French schools and a more present continuum must be created in learning programmes from the end of primary to the beginning of the secondary classes.

\section{1- Problématique}

Les compétences langagières orales, après avoir été le parent pauvre, voire oublié des Instructions officielles en France et des programmes d'étude au Québec, sauf pour l'école maternelle, occupent à présent une place honorable dans les écrits et les discours officiels. Depuis 1995, le ministère de l'Éducation du Québec (MÉQ) a enclenché une réforme de l'éducation dans laquelle l'oral retrouve sa place en tant que compétence disciplinaire, d'une part et en tant que compétence transversale, d'autre part, transcendant ainsi toutes les disciplines, du préscolaire à la fin du secondaire. L'évolution est identique en France à partir de 1992. Cependant, les outils qui permettraient d'aider les enseignants à rompre avec leurs pratiques et leurs représentations dans le domaine sont encore rares et 
de qualité scientifique inégale. Quant à la formation initiale des enseignants, elle privilégie davantage l'écrit.

Au-delà de ce statut nouveau de l'oral dans les textes officiels, il faut évoquer les enjeux sociaux d'un enseignement raisonné de l'oral. Il s'agit de distinguer (Dolz et Schneuwly, 1998; Nonnon, 1999; Le Cunff, 2000, sous presse; Lafontaine 2001; 2003) les différents aspects de l'oral. D'abord, l'oral est moyen de communication et, de ce point de vue, il est le support de tous les échanges qui se déroulent dans la classe. En conséquence, les productions de discours ont à voir avec les apprentissages dans toutes les disciplines. Les variétés, les situations discursives (Brossard, 1994) sont particulières à chacun des « épisodes » de la journée de classe. Ensuite, l'oral objet d'enseignement est une compétence - partie de la compétence langagière globale - qui fait l'objet d'une construction de la part de l'élève, continuée tout au long de la vie scolaire. En outre, les didactiques - en sciences notamment - ont montré que l'oral joue un rôle dans le domaine cognitif (Astolfi, 1986). Les théories relevant de l'interactionnisme social invitent également à considérer que les échanges participent à la construction des savoirs. La prise de parole au sein d'un groupe social - et la classe en est un parmi d'autres - est aussi ce qui permet de construire sa personne, d'exister par et pour les autres. C'est aussi la parole qui permet, au-delà de la dimension évidente de communication, de construire le groupe - le vivre ensemble - que ce soit au Québec ou en France. Toutes ces dimensions de l'oral mises en évidence par les travaux dans le domaine de la psychologie comme des sciences sociales et de la linguistique sont prises en compte dans la conception proposée par notre recherche pour construire une didactique raisonnée de l'oral.

\section{2- Cadre théorique}

La didactique générale s'incarne dans des disciplines spécifiques, comme la didactique des langues, en l'occurrence du français et, de façon plus précise ici, de l'oral. La linguistique reste la source première de la didactique des langues, mais il faut considérer ici une linguistique plus élargie qui accepte les discours oraux et écrits, l'énonciation et les variations sociales (Dabène, 1987). Ce constat semble appuyé par Simard (1997), qui affirme que l'enseignement de la langue maternelle doit entraîner une attitude sociolinguistique souple et ouverte face à tous les registres de langue afin d'échapper à la discrimination sur le langage, ce qui rejoint également les propos de Culioli (1973) affirmant que le langage, appréhendé à travers les âges, demeure un système ouvert.

Même si la didactique du français doit considérer l'enseignement de la langue écrite et de la langue orale, il ne faut pas passer sous silence l'appropriation des savoirs dans cette discipline. En effet, la scolarisation des savoirs présuppose une perception de l'enseignement du français dans laquelle l'objectif est la diffusion de savoirs savants (Halté, 1992). Halté (1992) précise que la didactique du français est divisée entre des savoirs à connotation culturelle et érudite et des savoirs savants et pratiques. C'est donc dire que le corps enseignant s'attend à ce que l'enseignement du français développe des savoirs utiles à toutes les disciplines, tel que le préconisent les programmes du 
MÉQ $(2001 ; 2003)$ et les Instructions Officielles en France. Le français demeurant la langue d'enseignement des autres matières scolaires, il est considéré comme un bien commun extérieur à l'école qui joue les rôles de socialisation et d'intégration dans la société par la culture et la langue. Selon Bronckart (1985), l'enseignement du français langue maternelle se distingue par deux types de finalités : finalités qui concernent les normes et finalités qui ont trait au fonctionnement langagier. Cet auteur précise également trois types d'objectifs qu'il croit réalisables en classe de français langue maternelle: des objectifs d'expression visant à amener l'élève à développer ses capacités, des objectifs de structuration visant à amener l'élève à observer les fonctionnements linguistiques et des objectifs d'adaptation visant à amener l'élève à s'adapter aux différentes situations qu'il devra affronter dans sa vie de francophone, tout comme le vise notre recherche.

Les sciences du langage imposent donc deux éléments cruciaux à l'enseignement du français: la communication orale et la culture de l'écrit. Lahire (1993) relève, dans l'histoire de l'enseignement de la langue maternelle en France, le constat suivant: dès 1872, avec Bréal, l'oral est au service de l'écrit. En effet, que ce soit le projet d'Instructions Rouchette (1963 à 1966), les Instructions Officielles de 1972, de 1977 à 1980 avec le Plan de rénovation Rouchette, l'enseignement du français a certes pour objet d'apprendre à l'élève à maîtriser l'expression orale, à quelques nuances près, mais son caractère fondamental est de donner accès à l'écrit.

La didactique de l'oral est donc une sous-division spécifique de la didactique de français. Elle est ouverte à tous les registres de langue, registres qui sont travaillés dans la classe de français langue maternelle à travers les activités d'oral pratiquées par les enseignants (MEQ, 1995, 2001, 2003; Simard, 1997). La didactique de l'oral est différente de la didactique de l'écrit, de la grammaire ou de la lecture, car elle s'intéresse à la fois au langage spontané de l'élève et au langage soutenu de celui-ci (Rigault, 1971; Boisseau et Raffestin, 1986; Doutreloux, 1989 ; Gadet, 1989, 1997; Charaudeau, 1992; de Pietro et Wirthner, 1996), tout comme le vise notre recherche au sujet de la prise de parole des élèves.

De plus, l'enseignement de l'oral n'a de sens et de probable efficacité que s'il est naturellement incorporé dans le milieu de vie et de communication qu'est la classe. En effet, une didactique de l'oral est centrée sur l'élève et peut lui offrir les connaissances et compétences nécessaires à la compréhension et à l'application d'un oral organisé que l'enseignant doit lui inculquer. Selon Doutreloux (1985) et Gadet $(1989 ; 1997)$, il faut sensibiliser l'élève de manière pratique et personnelle pour favoriser une amélioration de ses productions orales spontanées. D'autres chercheurs, tels que Tannen (1982), Snow (1983), Painchaud (1992), Ostiguy et Gagné (1992), Amétooyona (1995), de Pietro et Wirthner (1996) et Lafontaine $(2001 ; 2003)$ tiennent pour acquis que la didactique de l'oral doit également traiter de la langue décontextualisée, qui est la langue utilisée dans l'apprentissage des matières scolaires. De plus, les situations de communication présentées à la classe doivent être signifiantes et susciter un désir d'apprentissage chez les élèves. De cette façon, la 
communication orale engagera profondément l'apprenant et favorisera les circonstances d'interaction. Les élèves doivent sentir la pertinence du sens et des avantages des différents registres de langue des pratiques orales travaillées en classe. En bref, la didactique de l'oral doit adopter une démarche pédagogique réaliste respectant les besoins et aptitudes de chacun et s'orientant vers des objectifs sélectionnés à partir de besoins constatés par l'enseignant lors de productions orales réalisées en classe (Doutreloux, 1985; Wirthner, 1991), tout comme dans les activités observées chez les enseignants ayant participé à notre étude.

\section{3- Objectifs de recherche}

Notre recherche vise à valider ou à corriger des situations et des démarches isolées qui n'ont pu faire l'objet d'une expérimentation suffisamment large. Nous poursuivons l'objectif général suivant: mieux connaître les représentations des enseignants concernant l'oral et sa place dans les apprentissages comme dans la socialisation au sein de la classe quand ils mettent en place des situations innovantes. Nous traitons également trois objectifs spécifiques :

- Analyser en profondeur les situations et dispositifs mis en place pour les apprentissages visés;

- Évaluer les apprentissages effectués par les élèves dans le cadre des dispositifs choisis;

- Compte tenu des orientations respectives des Instructions Officielles en France et des programmes d'études au Québec, mettre en évidence les éléments de convergence ou de différence relatifs aux points qui intéressent la recherche menée en commun.

\section{4- Méthodologie}

Notre recherche, qui s'est déroulée de 2002 à 2004, s'inscrit dans le paradigme qualitatif et est exploratoire, car plusieurs éléments méthodologiques sont différents dans la mesure où deux échantillons sont issus de deux pays dans lesquels le traitement de la recherche avec des enseignants n'est pas organisé de la même façon, ce que nous approfondissons plus bas. Nous avons choisi les enseignants selon certains critères : leur intérêt pour l'enseignement de l'oral, leur connaissance des programmes d'études, leur intérêt pour la formation continue et leur réputation d'enseignants " experts " auprès de collègues, conseillers pédagogiques ou directions d'école. L'échantillon québécois est réduit à quatre enseignants et l'échantillon français à six enseignants ${ }^{1}$ afin de comprendre finement comment s'exerce la prise de parole en classe en lien avec la socialisation et les apprentissages des élèves dans chacun des pays. Il n'est donc pas possible de généraliser nos résultats. De plus, tel que démontré dans le tableau 1, certains enseignants ont effectué des activités d'oral avec le groupeclasse et d'autres, avec quelques élèves. Dans le cas d'activités comptant seulement quelques élèves, nous avons laissé les maîtres libres de choisir les participants. 
Nous avons d'abord voulu observer des activités d'oral en début d'année scolaire, alors que la prise de parole des enfants est encore « neuve » et, par la suite, les mêmes types d'activités d'oral en fin d'année scolaire afin de voir l'évolution des enfants et les représentations des enseignants au sujet de l'apprentissage des élèves en oral. Au Québec, l'étude s'est déroulée dans un milieu urbain : les régions de l'Outaouais et de la Montérégie. En France, la recherche s'est déroulée principalement en Bretagne et en Normandie, en milieux urbain et semi-urbain. Dans les deux pays, les écoles où les données ont été recueillies sont issues de milieux socio-économiques faible à élevé. Les élèves québécois sont tous des locuteurs natifs, c'est-à-dire que le français langue d'enseignement est leur langue première. En France, les élèves sont issus de milieux moyens ou défavorisés. Le français est généralement leur langue première pour ce qui concerne le primaire; au secondaire, certains élèves sont issus de l'immigration et une autre langue que le français est parlée à la maison. Les détails de chacune des activités se trouvent dans le tableau 1 et le contexte des pratiques de classe de chaque pays, en annexe. Nous expliquons à la suite de façon plus détaillées le contexte de notre recherche.

Tableau 1 : Caractéristiques des échantillons québécois et français

\begin{tabular}{|c|c|c|c|}
\hline Enseignants & $\begin{array}{l}\text { Ordres } \\
\text { d'enseignement }\end{array}$ & Activités filmées et durée & $\begin{array}{l}\text { Région et milieu } \\
\text { socio-économique }\end{array}$ \\
\hline $\begin{array}{l}\text { Enseignant } 1 \\
\text { Québec } \\
\text { (EQ1) }\end{array}$ & $\begin{array}{l}\text { Maternelle } \\
\text { (préscolaire) } \\
\text { Âge des élèves : } \\
5-6\end{array}$ & $\begin{array}{l}\text { Activité } 1 \text { : Causerie de } \\
\text { groupe animée par } \\
\text { l'enseignant sur la fête de } \\
\text { l'Halloween (durée : } 45 \\
\text { minutes) } \\
\text { Activité } 2: \text { Causerie de } \\
\text { groupe animée par } \\
\text { l'enseignant sur les insectes } \\
\text { (durée: } 45 \text { minutes) }\end{array}$ & $\begin{array}{l}\text { Outaouais } \\
\text { Milieu urbain } \\
\text { Revenu moyen }\end{array}$ \\
\hline $\begin{array}{l}\text { Enseignant } 2 \\
\text { Québec } \\
\text { (EQ2) }\end{array}$ & $\begin{array}{l}3^{\mathrm{e}} \text { année primaire } \\
\text { Âge des élèves : } \\
8-9\end{array}$ & $\begin{array}{l}\text { Activités } 1 \text { et } 2 \text { : Expérience } \\
\text { scientifique en petit groupe } \\
\text { de quatre enfants dirigée } \\
\text { par un élève expert et } \\
\text { retour sur l'activité en } \\
\text { grand groupe (durée : } 30 \\
\text { minutes par expérience } \\
\text { scientifique et } 20 \text { minutes } \\
\text { par retour en grand groupe) }\end{array}$ & $\begin{array}{l}\text { Montérégie } \\
\text { Milieu urbain } \\
\text { Revenus moyen à } \\
\text { élevé }\end{array}$ \\
\hline
\end{tabular}




\begin{tabular}{|c|c|c|c|}
\hline $\begin{array}{l}\text { Enseignant } 3 \\
\text { Québec } \\
\text { (EQ3) }\end{array}$ & $\begin{array}{l}5^{\mathrm{e}} \text { année primaire } \\
\text { Âge des élèves : } \\
10-11\end{array}$ & $\begin{array}{l}\text { Activité } 1 \text { : Discussion de } \\
\text { groupe animée par } \\
\text { l'enseignant sur la fête de } \\
\text { Noël et les traditions } \\
\text { familiales (durée : } 45 \\
\text { minutes) } \\
\text { Activité } 2 \text { : Discussion de } \\
\text { groupe animée par } \\
\text { l'enseignant sur le bilan de } \\
\text { l'année scolaire et les } \\
\text { vacances d'été (durée : } 45 \\
\text { minutes) }\end{array}$ & $\begin{array}{l}\text { Outaouais } \\
\text { Milieu urbain } \\
\text { Revenu moyen }\end{array}$ \\
\hline $\begin{array}{l}\text { Enseignant } 4 \\
\text { Québec } \\
\text { (EQ4) }\end{array}$ & $\begin{array}{l}1^{\mathrm{re}} \text { secondaire } \\
\hat{\text { Age des élèves : }} \\
12-13\end{array}$ & $\begin{array}{l}\text { Activités } 1 \text { et } 2: \\
\text { Discussion de deux } \\
\text { groupes de quatre élèves } \\
\text { sur divers contes (durée : } \\
40 \text { minutes par activité : } 20 \\
\text { minutes par groupe) }\end{array}$ & $\begin{array}{l}\text { Montérégie } \\
\text { Milieu urbain } \\
\text { Revenus moyen à } \\
\text { élevé }\end{array}$ \\
\hline $\begin{array}{l}\text { Enseignant } 1 \\
\text { France (EF1) }\end{array}$ & $\begin{array}{l}\text { Maternelle (petite } \\
\text { section) } \\
\text { Âge des élèves : } \\
2-4\end{array}$ & $\begin{array}{l}\text { Activités } 1 \text { et } 2: \\
\text { Discussion collective et } \\
\text { rituelle à visée } \\
\text { « scientifique » (durée : } 15 \\
\text { minutes par activité) }\end{array}$ & $\begin{array}{l}\text { Normandie } \\
\text { Milieu semi-urbain } \\
\text { Revenus faible à } \\
\text { élevé }\end{array}$ \\
\hline $\begin{array}{l}\text { Enseignant } 2 \\
\text { France (EF2) }\end{array}$ & $\begin{array}{l}\text { Maternelle } \\
\text { (grande section) } \\
\text { Âge des élèves : } \\
5-6\end{array}$ & $\begin{array}{l}\text { Activité } 1: \text { Jeu rituel, objet } \\
\text { à découvrir (durée : } 20 \\
\text { minutes) } \\
\text { Activité } 2: \text { Discussion en } \\
\text { groupe } \\
\text { (durée : } 20 \text { minutes) }\end{array}$ & $\begin{array}{l}\text { Banlieue éloignée et } \\
\text { rurale de Paris } \\
\text { Milieu semi-urbain } \\
\text { Revenus faible à } \\
\text { élevé }\end{array}$ \\
\hline $\begin{array}{l}\text { Enseignant } 3 \\
\text { France (EF3) }\end{array}$ & $\begin{array}{l}1^{\mathrm{re}} \text { année } \\
\text { primaire } \\
\text { Âge des élèves : } \\
6-7\end{array}$ & $\begin{array}{l}\text { Activité } 1 \text { : Présentation en } \\
\text { groupe (science, durée : } 30 \\
\text { minutes) } \\
\text { Activité } 2 \text { : Discussion } \\
\text { collective (science, durée : } \\
30 \text { minutes) }\end{array}$ & $\begin{array}{l}\text { Normandie } \\
\text { Milieu urbain } \\
\text { Revenus faible et } \\
\text { moyen }\end{array}$ \\
\hline $\begin{array}{l}\text { Enseignant } 4 \\
\text { France (EF4) }\end{array}$ & $\begin{array}{l}2^{\mathrm{e}} \text { année primaire } \\
\text { Âge des élèves : } \\
7-8\end{array}$ & $\begin{array}{l}\text { Activité } 1: \text { Observation en } \\
\text { groupe (technologie, } \\
\text { durée : } 30 \text { minutes) } \\
\text { Activité } 2: \text { Présentation en } \\
\text { groupe (technologie, } \\
\text { durée : } 30 \text { minutes) }\end{array}$ & $\begin{array}{l}\text { Normandie } \\
\text { Milieu urbain } \\
\text { Revenus moyen et } \\
\text { faible }\end{array}$ \\
\hline $\begin{array}{l}\text { Enseignant } 5 \\
\text { (EF5) } \\
\text { France }\end{array}$ & $\begin{array}{l}3^{\mathrm{e}} \text { année primaire } \\
\text { Âge des élèves : } \\
8-10\end{array}$ & $\begin{array}{l}\text { Activités } 1 \text { et } 2: \text { Débat } \\
\text { collectif (durée : } 30 \\
\text { minutes) }\end{array}$ & $\begin{array}{l}\text { Normandie } \\
\text { Milieu rural } \\
\text { Revenu moyen }\end{array}$ \\
\hline
\end{tabular}




\begin{tabular}{|l|l|l|l|}
\hline $\begin{array}{l}\text { Enseignant 6 } \\
\text { (EF6) }\end{array}$ & $\begin{array}{l}2^{\mathrm{e}} \text { secondaire } \\
\text { Arge des élèves : } \\
\text { France }\end{array}$ & $\begin{array}{l}\text { Activité 1 : Création d'un } \\
\text { poème collectif (durée : 25 } \\
\text { minutes) }\end{array}$ & $\begin{array}{l}\text { Bretagne } \\
\text { Milieu urbain } \\
\text { Revenu faible }\end{array}$ \\
& $\begin{array}{l}\text { Activité 2 : Analyse d'un } \\
\text { texte de théâtre (durée : 25 } \\
\text { minutes) }\end{array}$ & \\
\hline
\end{tabular}

\section{1- Contexte de la recherche}

Notre recherche a pris son envol dans le cadre d'un projet plus large, celui d'un échange professionnel d'enseignement/recherche entre nous, chercheures. $\grave{A}$ la suite de ce projet, nous avons chacune rédigé un rapport de recherche pour nos établissements faisant état des résultats québécois pour Lizanne Lafontaine et des résultats français pour Catherine Le Cunff. Nous avons également comparé les résultats entre les deux pays dans chacun des rapports. Dans cet article, nous évoquons le côté "recherche" du projet et présentons une synthèse de nos rapports $^{2}$.

Deux collectes de données ont eu lieu dans les deux pays : une à la phase 1 et l'autre, à la phase 2 . Dans la phase 1 du projet, Lizanne Lafontaine (chercheure québécoise) a reçu Catherine Le Cunff (chercheure française) dans son institution, l'Université du Québec en Outaouais, pendant six semaines, soit du début octobre à la mi-novembre 2002 . Pendant ce séjour, nous avons recueilli les premières données dans les classes québécoises et effectué les entretiens auprès des enseignants. Les données françaises (activités de classe et entrevues auprès des enseignants) avaient été recueillies par Catherine Le Cunff avant son séjour au Québec et en décembre, à son retour.

Dans la phase 2 du projet, Catherine Le Cunff a accueilli Lizanne Lafontaine dans les murs de son institution de l'époque, l'Institut de formation des maîtres de Bretagne, pendant huit semaines, soit de la fin mars à la mi-mai 2003. Pendant cette période, nous avons recueilli les secondes données françaises dans les classes et réalisé les seconds entretiens avec les enseignants français. À son retour de France, en juin 2003, Lizanne Lafontaine a collecté les dernières données du Québec et a fait les dernières entrevues avec les enseignants.

Finalement, la phase 3 de la recherche s'est réalisée de septembre 2003 à août 2004 où nous nous sommes rencontrées à deux reprises, en mars et en août, afin de terminer l'analyse des données et de diffuser nos résultats au colloque de l'Association internationale de recherche en didactique du français (AIRDF) qui s'est tenu à Québec, en août 2004.

Il est maintenant plus facile de comprendre que les données de notre recherche ont été parfois recueillies par les deux chercheures et parfois, par la chercheure dans son pays d'origine. Chacune des chercheures a également analysé ses propres corpus afin de respecter les cultures scolaires (systèmes éducatifs, programmes d'études, formation des enseignants) et méthodologiques (équipes de recherche, exigences institutionnelles). Cette façon de faire n'a pas biaisé les résultats, car nous avons suivi les procédures méthodologiques que 
nous nous étions fixées avec rigueur, ce que nous expliquons à la prochaine section.

\subsection{Collecte et analyse des données}

Nous rappelons que la collecte des données s'est effectuée en deux temps dans les deux pays. D'abord, entre les mois de septembre et de décembre 2002, nous avons filmé une première activité d'oral dans les classes des enseignants dans lesquelles nous faisions une observation non participante. Ces corpus ont été transcrits. Par la suite, des entrevues semi-dirigées enregistrées sur cassette audio ont été réalisées avec les enseignants. Les entretiens visaient essentiellement à recueillir les impressions des enseignants au sujet de l'activité filmée dans leur classe et à connaître leurs perceptions d'éléments fondamentaux en enseignement de l'oral qui ont pu appuyer les constats issus de nos analyse de données.

La deuxième collecte des données a eu lieu entre les mois de mars et de juin 2003. Nous sommes retournées dans les mêmes classes pour filmer le même type d'activité d'oral qu'au début de l'année scolaire et en jouant encore le rôle d'observatrices non participantes. Les corpus ont également été transcrits. Nous avons aussi effectué d'autres entretiens semi-dirigés avec les enseignants québécois et français afin de recueillir leurs observations au sujet de l'évolution de la prise de parole de leurs élèves tout au long de l'année scolaire. Nous rappelons que le tableau 1 fait état des activités filmées dans les deux pays.

Afin d'analyser nos données, nous avons utilisé la méthode d'analyse de contenu par catégories. Les catégories d'analyse ont été élaborées à la suite de plusieurs échanges et de notre première rencontre lors du séjour de Catherine Le Cunff au Québec. Lors du séjour de Lizanne Lafontaine en France, nous avons validé nos catégories en analysant les transcriptions de la première activité des enseignants EQ1 et EF2 (maternelle 5-6 ans). Les catégories d'analyse retenues ont été les suivantes: distinctions entre les ordres d'enseignement, place de l'adulte dans les activités orales, nombre de prise de parole, séquences discursives, stratégies de prise de parole des élèves, prise en compte de l'autre et socialisation. Finalement, dans chacun des pays, les enseignants ont été partie prenante du processus d'analyse, car ils ont validé nos analyses. Nous rappelons que chaque chercheure a analysé ses corpus pour les raisons évoquées plus haut. Toutefois, comme nous avons régulièrement croisé nos analyses entre chercheures lors de nos visites et échanges par courrier électronique et que nous avons confronté nos analyses à celles des enseignants participants, nous avons pu trianguler nos données et ainsi éviter les biais.

\subsection{Différences méthodologiques}

Le déroulement de la recherche a été le même au Québec et en France : observations non participantes filmées d'activités d'oral dans les classes, entretiens avec les enseignants, analyse des données par catégorie et triangulation. Nous avons ainsi respecté le protocole de la recherche qualitative. Par contre, comme le Québec ne dispose pas de structure de recherche permettant le dégagement des enseignants pour devenir praticiens-chercheurs 
comme c'est le cas en France, nous n'avons pas pu constituer d'équipe de recherche officielle comme l'a fait Catherine Le Cunff. Le contexte culturel ne nous l'a pas permis, ce qui n'a pas empêché le travail en duo avec les enseignants québécois pour valider les analyses de Lizanne Lafontaine afin d'atteindre les objectifs de l'étude.

Deux autres éléments différents sont les programmes d'études et le système éducatif de chaque pays et ont nécessité une analyse unique selon le pays d'origine des chercheures, comme nous l'avons expliqué précédemment. Ces aspects n'ont pas nui à la réalisation de la recherche, ils sont plutôt devenus un apport comparatif intéressant et nous ont permis de répondre à notre objectif spécifique 3. Comme le Québec se trouve en pleine réforme de l'éducation, privilégiant le paradigme de l'apprentissage au lieu du traditionnel paradigme de l'enseignement, il a été intéressant de comparer les résultats français et québécois.

\section{Résultats comparatifs de l'analyse des données}

Nous présentons les résultats de l'analyse comparative entre les échantillons québécois et français. Nous tentons de démontrer comment nous avons pu atteindre nos objectifs de recherche, et ce, malgré les différences méthodologiques que nous venons d'évoquer. Pour ce faire, nous proposons plusieurs extraits de corpus québécois et français faisant état de nos catégories d'analyse : distinctions entre les ordres d'enseignement, place de l'adulte dans les activités orales, nombre de prises de parole, séquences discursives, stratégies de prise de parole des élèves, prise en compte de la parole de l'autre et socialisation.

\subsection{Distinctions entre les ordres d'enseignement}

Tel qu'expliqué dans la section précédente, nous avons observé de nettes distinctions entre le préscolaire/primaire et le secondaire. D'abord, au préscolaire/primaire québécois, l'oral est intégré à la vie de la classe et aux autres volets du français. Il y a métacommunication et mobilisation de la compétence transversale de la communication. De plus, il y a pratique régulière formelle et informelle de l'oral en classe, pratique qui respecte le contenu et les objectifs du programme d'études du MÉQ (2001).

$\mathrm{Au}$ secondaire québécois, nous avons constaté que l'oral n'est pas intégré à la vie de la classe et aux autres volets du français. La pratique de l'oral est irrégulière, car elle est seulement sommative (deux fois par année). Le programme d'études du français au secondaire en vigueur lors de la collecte des données (MÉQ, 1995) est respecté dans le genre, mais pas dans la façon de concevoir l'oral en classe. De plus, nous avons remarqué que la prise en charge de la prise de parole des élèves est faite de façon implicite, surtout chez les plus forts, car l'oral n'est pas enseigné. La reformulation en tant que stratégie de prise de parole est trop peu utilisée, même si elle est un outil métacognitif important.

Nous avons également constaté une volonté de changement chez les enseignants québécois même si plusieurs ne savent pas comment considérer 
l'oral comme objet d'enseignement raisonné et qu'ils disent manquer d'outils. Cela est très intéressant et rejoint nos objectifs de recherche.

\subsection{Place de l'adulte dans les activités}

Nous avons noté des différences intéressantes entre la façon dont les enseignants québécois et français considèrent la place de l'adulte dans les activités orales. D'abord, dans les activités au préscolaire/primaire au Québec, nous avons remarqué que l'adulte occupe beaucoup de place: il fait un étayage, il dirige la prise de parole ou les rôles, il reformule à la place de l'élève sans le consulter et il complète souvent la phrase de l'élève en parlant pour lui. En voici un exemple :

Québec : extrait corpus primaire $\mathrm{EQ}^{3}$ (voir annexe)

A : O.K., bien moi naturellement à chaque année je fête un Noël traditionnel. Comme les seules lumières qui éclairent la salle, c'est les lumières dans l'arbre de Noël puis une lampe de chevet, puis on déballe les cadeaux puis on arrête à moitié chemin on va changer. Mais j'aimerais ça aussi que ça change, puis que ça soit une pièce un peu plus éclairée, qu'on mange comme pas nécessairement plus tard, mais plus tôt à la place, comme avant de déballer les cadeaux. Puis qu'on n'ait pas seulement de la dinde puis de la tourtière parce que je commence à être un peu tannée.

$E: O . K$., fait que tu aurais d'autres désirs au sujet de la façon dont ça se passe. $C ?[\ldots]$

$J$ : Moi bien moi je passe deux Noël, à cause que un chez ma mère puis un chez mon père. Le Noël chez ma mère c'est ... mon frère invite ses amis puis ma grande scur aussi puis on fait ... on fait ... on mange pas de dinde, mais on fait un genre de gros souper puis on déballe nos cadeaux ...

E: Dans la soirée?

$J:$ Oui.

$J:$ Puis avec mon père, bien il invite ses frères et ses scurs...

$E$ : Puis là il y a une grosse fête. O.K., tu veux-tu que ça reste encore comme ça cette année? Tu n'as pas de désir spécial? Non? Tout est correct? O.K.. M?

Nous constatons que l'enseignant termine la phrase de l'élève «Puis là il y a une grosse fête ». Il parle donc à la place de l'élève. Du côté des enseignants français, l'élève occupe davantage la place qui lui revient, comme en fait foi cet extrait.

France : extrait corpus primaire EF5 (voir annexe)

$E$ : Effectivement vous avez tous été touchés par la marée noire donc c'est le sujet qu'vous avez choisi ... Mais ça veut pas dire que les autres on les traitera jamais ... On les traitera p't être à un moment donné ... Alors pourquoi A je l'ai mise au centre ... là-bas comme une grande (rire)? Ben?

Ben : Parc'que c'est la présidente.

$E$ : C'est la présidente ... elle a quel rôle ? ... Ju?

$J u$ : Ben elle doit pas parler 
$E:$ Ah bon ? ... elle doit pas parler du tout?

Ju: Mais euh ... quand quelqu'un par exemple lève la main et ben elle dit euh oui par exemple levez la main

$E$ : Alors c'est elle qui fait quoi?

$J u:$ Ben c'est elle la maîtresse?

$E$ : Pas tout à fait .......Ju?

Ju C'est elle qui décide qui va parler.

E: Oui.

Ca: Qui mène le débat.

$E$ : C'est elle qui mène le débat ... D'accord? ... Donc vous ne pouvez pas parler ... sans son autorisation ... Vous levez la main ... et quand A désigne qui veut parler ... c'est bon vous y allez ... D'accord? ... Le secrétaire il a quel rôle? Flo ? ... Ju

Avant de laisser les élèves débattre du thème qu'ils ont choisi eux-mêmes, l'enseignant EF5 explicite son rôle, celui du président et du secrétaire. Les modalités d'intervention dans le débat «donner son opinion » sont également clarifiées, car les élèves ne sont pas familiers avec ce type de discours et pensent qu'il s'agit de questionner et de répondre à des questions, forme d'échange ordinaire à l'école. Nous avons ici un très bon exemple de la volonté des enseignants français de laisser l'espace sonore aux élèves et de n'intervenir que pour réguler, étayer une prise de parole qu'ils veulent autonome, ce qui, nous l'avons vu, n'est pas toujours le cas au Québec.

\subsection{Nombre de prises de parole}

Il est très intéressant d'observer que dans le corpus québécois du préscolaire/primaire, nous avons dénombré 178.4 prises de parole pour 93 élèves dans des activités ayant une durée de 45 minutes, soit une moyenne par élève de 10.3 prises de parole. C'est très peu compte tenu du nombre d'élèves. De plus, les tours de parole étaient majoritairement sollicités au préscolaire et en $5^{\mathrm{e}}$ année (activités dirigées par les enseignants), mais étaient spontanés en $3^{\mathrm{e}}$ année (discussion entre élèves). Nous pouvons faire un lien direct avec la place de l'adulte dans la prise de parole évoquée à la section précédente. Voici un extrait de corpus en faisant foi :

Québec : extrait corpus préscolaire EQ1 (voir annexe)

$E$ : Je vais te le dire, j'ai choisi des dessins rigolos parce que je sais que peutêtre il y en a qui ont peur. Alors je voudrais savoir qui n'aime pas les monstres épeurants? Les masques très laids? O.K. Alors ne vous en faites pas, il n'y en aura pas. D'accord, F? D'accord, J? Mais, mais $C$ tu as le droit d'aimer ça et je peux peut-être trouver une solution pour toi. Quel atelier pourrait contenir des monstres épeurants? Y a-t-il un atelier ... as-tu une idée $C$ ? $C$ et $C D$, tu $m$ 'as dit bon bien ...

C. : On pourrait ...

E : $\quad$ Où est-ce qu'on pourrait en mettre des monstres épeurants?

$C D$. : À côté ... bien en haut de le monstre. 
E: $\quad$ Oui, sur mon tableau ici d'ailleurs, mais dans la classe là pour que $C$ puisse choisir un coin de jeux où il y aurait des monstres épeurants, où est-ce que je pourrais en mettre, $X$ ?

$X: \quad$ Au coin maison!

E: $\quad$ C'est parce que, mon amour, ça serait une bonne idée si tout le monde aimait les monstres épeurants, mais plusieurs amis ont peur fait que je pense qu'on est mieux de ne pas avoir de monstres épeurants au coin maison. Est-ce $q u ' i l y$ a un autre endroit?

$X:$ Ah, moi je le sais!

E : Écoutez! Ferme tes yeux deux petites minutes! Ferme tes yeux, je te regarde. Pense dans ta tête les yeux fermés aux différents coins de jeux que nous avons, je vais en nommer ... Ah! Je n'ai pas besoin d'en nommer? Pense aux coins de jeux et dis-moi où est-ce que quelqu'un pourrait travailler avec des monstres épeurants. $\mathrm{F}-\mathrm{O}$ ?

$\mathrm{F}-\mathrm{O}$ : Les maisonnettes.

E: $\quad$ Si on trouvait des petits monstres épeurants pour mettre au coin de maisonnettes?

F-O: Oui!

$E$ : $\quad$ D'accord! Quelqu'un a une autre idée? F mon amour! Vas-y!

$F:$ On pourrait ajouter au coin maison un vampire!

E : $\quad$ Est-ce qu'il a le droit d'être épeurant?

Plusieurs: Non.

$E$ : $\quad$ Non, pourquoi $F$ ?

$F: \quad$ Ily a quelques amis qui ont peur. [...]

E : Oui, c'est doux, tu as raison, oui, c'est doux! C'est une sorte de tissu les amis, c'est un matériel qu'on utilise pour faire des marionnettes comme $M$ disait, on peut faire beaucoup de bricolage, et tu vois là le magasin, lui, il m'en a fait en forme d'animaux, mes sapins, tu te souviens comme $F$ disait, $F$ aime beaucoup faire des suites avec les objets fabriqués en feutrine. Alors on pourra pour $C$ prendre de la feutrine et fabriquer des personnages épeurants. V lève la main! On a un autre petit quatre minutes et après on finit!

Toutefois, au secondaire au Québec, le nombre de prises de parole est beaucoup plus élevé pour moins d'élèves et pour une durée plus courte. Les huit élèves participants ont fait 377.5 prises de parole, soit une moyenne de 47.2 prises de parole par élève. De plus, les tours de parole étaient majoritairement spontanés, car ils étaient dirigés par les élèves.

Il est donc intéressant de voir que huit élèves de $1^{\mathrm{re}}$ secondaire s'expriment plus, dans une activité plus courte (deux fois 20 minutes) dirigée par les élèves que dans la classe du préscolaire et les deux classes du primaire observées.

France : extrait corpus préscolaire EF2 (voir annexe)

E : Lu apporté une devinette .... Comment se passe ce jeu? ... Qui est ce qui parle?

Clé : Faut poser des questions.

$E$ : Alors Clé tu nous expliques ... vas-y. 
Clé : Il faut poser des questions.

$E$ : Qui pose des questions? ... les enfants ou Lu?

Plusieurs élèves (ensemble) : NOUS!

$E:$ À qui vous posez des questions?

Plusieurs élèves (ensemble) : à Lu!

Clé : Lu il nous pose aussi des questions pour nous dire ... c'est pas ça ... ou c'est ... ou c'est ça.

El: Non c'est pas ça.

$E:$ Lu ... il vous pose aussi des questions ? ... Vous êtes d'accord avec ça?

Quelques élèves: Non.

$E: J-V i$... Lu vous pose aussi des questions?

$G: C$ 'est nous!

$E: O u i ?$

$G:$ Nous on dit ... Nous on dit ...

E:Tu attends.

$G:$ Lu nous on dit des questions ... et lui ... et lui il doit nous répondre par une phrase ... c'est OUI ou NON.

$E$ : Nous on pose une question ... on demande quelque chose à $\mathrm{Lu}$... et $\mathrm{Lu}$ doit nous répondre par une phrase ... c'est très bien ...?

El : Il dit OUI ou NON.

E : Il nous répond par OUI ou par NON et il fait une belle phrase derrière ... D'accord? ... Tu te rappelles de la règle du jeu Lu ? ... Bon ... À quoi servent vos questions? ... À quoi servent vos questions?

Plusieurs élèves : À gagner !... À gagner!

Cin (tout doucement) : $A$ trouver.

$E$ : Cin vas-y ... à quoi servent nos questions ma puce?

Cin : $A$.... deviner c'que c'est.

Cet extrait est représentatif de l'objectif des enseignants français du préscolaire comme du primaire. Avant de donner la responsabilité progressivement de l'échange oral aux élèves eux-mêmes, ils clarifient la tâche langagière, se situant donc au plan métalinguistique avant de laisser les élèves occuper l'espace sonore, comme nous l'avons vu plus tôt. Leur rôle est d'étayer et de réguler afin que les apprentissages qui se font au cours de ce « jeu » s'effectuent. Ils laissent ainsi plus de place aux élèves. Toutefois, cela semble fort différent au secondaire français où l'oral constitue une forte prise de risque de débordement dans les collèges et se limite donc à une forme ordinaire de l'échange questionréponse, sauf quand le débat, par exemple, est explicitement travaillé d'un point de vue technique.

\subsection{Séquences discursives}

Aux trois ordres d'enseignement, les séquences discursives étaient narratives, descriptives et explicatives. Nous remarquons aussi que les séquences explicatives n'étaient souvent pas abouties, autant en France qu'au Québec. Nous pouvons faire le lien avec l'enseignement donné en classe concernant le texte explicatif, par exemple. Au Québec, ce type de texte est enseigné de façon 
systématique en troisième secondaire. Toutefois, les élèves sont sensibilisés aux séquences explicatives dès le préscolaire. Nous pouvons penser que cette sensibilisation énoncée dans les programmes d'études du MÉQ a des répercussions négatives dans la prise de parole des enfants parce qu'ils n'arrivent pas souvent au bout de leur explication.

Québec : extrait corpus préscolaire EQ1 (voir annexe)

$E$ : Qu'est-ce que tu connais par rapport aux insectes? Oui, $M$.

$M: L$ 'abeille.

E : $\quad$ Toi tu connais l'abeille ... Un instant! ... Tu lèves si tu veux me parler mon amour! (silence) Est-ce que quelqu'un d'autre veut me dire quelque chose? $C$ : Une couleuvre...

E: $\quad$ Toi quand on parle des insectes ça te fait penser à une couleuvre? Peux-tu me dire pourquoi?

$C$ : Parce que j'ai déjà vu une couleuvre à ma garderie.

E: $\quad T u$ as déjà vu une couleuvre à ta garderie? O.K., mais pourquoi quand je te parle des insectes ça te fait penser à la couleuvre?

$C$ : C'est juste comme... quand j'ai vu une couleuvre à ma garderie, c'est pour ça.

Nous pouvons observer dans cet extrait du préscolaire que l'explication de $\mathrm{C}$ au sujet de la couleuvre étant un insecte n'est pas aboutie. L'enseignant n'amène pas l'élève à expliquer davantage pourquoi le fait d'avoir vu une couleuvre à la garderie la place dans la famille des insectes.

France : extrait corpus préscolaire EF1 (voir annexe)

$O:$ Des noisettes et de la salade.

$B:$ Ah oui les chats mangent de la salade.

$E$ : Les chats mangent de la salade.

$O:$ NON ... non c'est pas vrai, j'ai jamais vu.

$E$ : Non c'est pas vrai ... Lu tu es sûr de ça? Alors attendez j'ai une question à poser à $L u$... Lu est-ce que tu as un chat?

Lu: Euh oui mais il est mort.

$E$ : Il est mort ... alors on ne peut pas en parler ... qui a un chat dans sa maison?

$B:$ Moi.

$E: B \ldots$ B est ce que ton chat ...

Do: $M O I !$

$E$ : Je parle à $B$ s'il te plaît.

$B: M o i$, mon chat mange du poisson.

$E$ : Ton chat mange du poisson. Attends j'ai une autre question. Est-ce que ton chat mange des noisettes?

$B:$ Non

$E:$ Non ... Est-ce que ton chat mange de la salade?

$B:$ Non. 
$E:$ Non ... euh ... qui encore a un chat $? V \ldots V \ldots$ Est-ce que ton chat mange des noisettes?

$V:$ Non.

E: Est-ce que ton chat mange de la salade?

$V:$ Non.

Dans cette classe française, les enfants ont entre 2 et 3 ans 6 mois. Ils travaillent chaque jour pendant un court moment à constituer une fiche d'identité pour les animaux familiers. Ils disent ce qu'ils savent, discutent et lorsqu'ils ne sont pas d'accord, doivent demander aux parents. L'enseignant a pour objectif de construire avec eux une démarche de l'ordre du scientifique, de la découverte active du monde en permettant une confrontation des idées. L'un d'entre eux a dit que les chats mangent des noisettes, d'où la discussion. Nous constatons que l'enseignant EF1 travaille les séquences discursives: il cherche à faire produire un discours de type explicatif.

\section{Stratégies de prise de parole}

$\mathrm{Au}$ préscolaire/primaire québécois, les stratégies de prise de parole sont beaucoup de l'ordre de la question-réponse à l'enseignant dans les activités de maternelle (EQ1) et de $5^{\mathrm{e}}$ année (EQ3), car dans ces activités, c'est l'enseignant qui dirige la discussion. Toutefois, dans ce type de stratégie, nous avons remarqué une sous-stratégie très coquine dans la classe de maternelle du Québec: verbaliser sa réponse à l'autre afin qu'il la donne à l'enseignant. Par exemple, une élève qui prenait beaucoup la parole n'était plus questionnée par l'enseignant. Pour pouvoir continuer à participer à la discussion, elle chuchotait dans l'oreille de son amie la réponse, levait bien haut le bras de sa copine qui, questionnée par le maître, verbalisait la réponse. Celle-ci pouvait ainsi donner son idée même si elle ne parlait pas. Nous pouvons penser que cette élève, malgré son jeune âge, a bien compris le pouvoir de la parole et la façon de la prendre autrement afin de s'exprimer.

Les autres stratégies de prise de parole au primaire sont directement en lien avec les conduites discursives exigées par l'activité.

Québec : extrait corpus primaire EQ2 (voir annexe)

G (animateur) : $\quad \ldots . O . K$. tu le fais, O.K., c'est correct là tu le mets dans un des verres. Mets-le dans celui-là, bon là on mélange pas de tout de suite, mais tu le laisses ... on va attendre ... on va attendre ... Là qu'est-ce que vous voyez? Qu'est-ce que ça fait?

$P$ : Bien ...

$G$ : $\quad$ Bien non fais-le pas tout de suite, il faut pas que tu le fasses tout de suite, mets ça dans le fond.

$P: \quad \quad$ O.K. je remets ça dans le pot.

$P a: \quad O u i$, on remet ça dans le chose...

$E: \quad$ Le fond est plein, le fond est blanc.

$G: \quad O . K$. là dans le volume page 17, vous voyez en bas là ... réalisation de $A$, vous voyez Eau: avant de remuer les mélanges ... sucre qu'est-ce que ça 
fait? Vous allez dessiner dans le bas A. Vous allez mettre une barre pour voir la hauteur de l'eau, vous allez comme colorier pâle dans le fond pour voir que le sucre ... et le bicarbonate de soude... est-ce que vous avez tout fait? Bon, O.K.. O.K., bicarbonate de soude ... qui qui s'occupe de ça le bicarbonate...

Il s'agit de l'expérience scientifique vécue en $3^{\mathrm{e}}$ année. Dans cette activité, l'animateur (Gilles) a utilisé les stratégies suivantes: questionner ses coéquipiers, donner des consignes, suivre le plan de l'expérience et gérer les tours de parole (étayage fait par l'enfant). Les participants à l'activité suivaient ce que leur disait l'animateur.

Au secondaire québécois, les élèves ont également utilisé des stratégies en lien avec les conduites discursives exigées par l'activité de la discussion. Ils ont suivi à la lettre le plan de la discussion, ce qui, comme nous l'avons observé plus tôt, les a empêchés de réellement discuter. Ils ont également posé quelques questions. Une autre stratégie a été d'appuyer ce que l'autre a dit, d'interrompre l'autre afin de donner son idée et de reformuler. Toutefois, la reformulation, qui nous semble une stratégie très intéressante, a été très peu utilisée au Québec, contrairement en France, et nous semble une piste de recherche intéressante.

En somme, il est normal que les élèves québécois du secondaire utilisent davantage de stratégies de prise de parole, car leur expérience de locuteur est plus grande. Cependant, comme la prise de parole n'est pas enseignée de façon systématique en classe, elle demeure souvent intuitive et plus élaborée chez les élèves plus forts, qui sont souvent plus éloquents.

\subsection{Prise en compte de la parole de l'autre}

Nous avons constaté que la prise en compte de la parole de l'autre est difficile au préscolaire/primaire québécois, surtout du préscolaire à la $3^{\mathrm{e}}$ année. En effet, l'enseignant devait faire des rappels à l'ordre fréquents au préscolaire. Il était difficile pour l'animateur en $3^{\mathrm{e}}$ année de prendre conscience de la parole de l'autre, car il devait se concentrer sur son rôle, et ce, dans une activité nouvelle. Ces enfants étaient jeunes pour jouer de tels rôles. Au secondaire, nous avons cependant remarqué que la prise en compte de la parole de l'autre s'avérait facile pour l'animateur.

Malgré ces différences, les élèves de tous les niveaux, en France et au Québec, même s'ils étaient parfois fortement dirigés par les enseignants au Québec (EQ1 et EQ3), respectaient les tours de parole, étaient attentifs à l'autre, s'appuyaient sur la parole de l'autre, et ce, à différents stades selon les âges. En France, le travail sur la prise en compte de la parole de l'autre est le résultat d'une évolution voulue par les enseignants. Ces constats nous amènent à proposer des pistes de recherche quant à l'écoute et à la compréhension orale dans des situations de prise de parole en groupe.

\subsection{Socialisation}

La socialisation joue un rôle important dans la prise de parole. Par exemple, au préscolaire au Québec, les élèves exprimaient un message en je afin de dire à un camarade qu'il les dérangeait; en $3^{\mathrm{e}}$ année, l'animateur s'occupait 
de l'élève turbulent. Au secondaire, les élèves s'encourageaient à s'exprimer les uns les autres et ont appris à recevoir et à donner de la critique par l'évaluation par les pairs qui était donnée en fin de séance. À tous les niveaux, les désaccords étaient exprimés de façon polie; quelques élèves interrompaient, mais ils respectaient l'autre.

Québec : extrait corpus préscolaire EQ1 (voir annexe)

$Z:$... Lâche-moi $C$ de me donner des coups! S'il te plaît ça me dérange!

E : $\quad O . K$., tu as compris mon $C$ ? O.K., $Z$, bouge plus et tu vas être en parfait état. Bon ben les amis, on va faire la même activité qu'on avait faite à l'automne.

Québec : extrait corpus $1^{\mathrm{re}}$ secondaire EQ4 (voir annexe)

$S$ : (rire) O.K. Bien moi je m'appelle $S$, bien j'ai trouvé qu'ils étaient bien préparés puis qu'ils avaient donné des bonnes explications aux questions. Parce que des fois $J F$, il riait, bien souvent il riait, puis il parlait vite un peu, mais ils étaient bien préparés. Puis il y a eu quelques moments de silence, mais c'était bon en général.

$C$ : Moi c'est $C$, ben je trouve qu'ils ont tous bien participé puis qu'ils ont donné des bons commentaires puis tout ça. Mais c'est aussi bien c'est ça JF il riait beaucoup. Ben ... E elle ne parlait pas assez fort! Puis c'est ça, mais à part ça je trouve que c'était bien préparé là, puis que c'était intéressant.

An: Moi c'est An... bien ... moi je dis que ... F et JF ont parlé beaucoup et ils ont laissé la place aux autres. Mais les autres ne parlaient pas beaucoup peutêtre et ils ne parlaient même pas assez fort. Il y avait beaucoup de moments de silence.

\section{Discussion}

À la lumière de nos analyses, nous croyons que nous avons atteint nos objectifs de recherche. En effet, notre analyse a pu nous permettre de mieux connaître les représentations de certains enseignants français et québécois concernant l'oral et sa place dans les apprentissages comme dans la socialisation au sein de la classe quand ils mettent en place des situations innovantes (objectif général). En ce sens, il est ressorti de nos résultats que l'oral doit être enseigné de façon systématique à l'école au Québec et en France et qu'un continuum plus présent doive se faire sentir dans les programmes d'études entre la fin du primaire et le début du secondaire. L'arrimage entre ces deux ordres d'enseignement est effectivement peu étudié et le fossé identifié entre les pratiques d'oral au préscolaire/primaire et au secondaire se creuse encore malgré les réformes des programmes.

Ensuite, nous avons pu répondre à nos objectifs spécifiques. D'abord, nous avons analysé en profondeur les situations et dispositifs mis en place pour les apprentissages visés (objectif spécifique 1) et évalué les apprentissages effectués par les élèves dans le cadre de ces dispositifs (objectif spécifique 2). 
La reformulation, outil de métacognition et de socialisation, est trop peu travaillée à l'école québécoise. Dans le même ordre d'idées, puisque l'écoute et la compréhension orale étant préalables à une production orale de qualité, il importe de leur donner la place qu'il leur revient dans les pratiques de la classe, les programmes ministériels et dans les programmes de formation initiale des maîtres. Nous avons aussi observé que les élèves du secondaire québécois prennent davantage la parole et plus facilement que les élèves du primaire, mais souvent de façon plus brève et moins structurée, parfois inutile. En d'autres mots, même si les élèves du secondaire s'expriment plus, ils ne font pas toujours évoluer l'argumentation ou la discussion. Cela peut être dû au fait que l'enseignant EQ4 n'a pas enseigné l'oral en classe, mais a plutôt fait deux activités sommatives sans préparation. Les élèves n'ont que répondu à des questions.

De plus, tout au long de notre article, nous avons réussi à mettre en évidence les éléments de convergence ou de différence des pratiques québécoises et françaises au regard des programmes ministériels de chacun des pays (objectif spécifique 3). Nous pouvons ainsi affirmer que l'oral tel que présenté ici illustre bien le paradoxe dans lequel se trouvent les enseignants de français québécois du secondaire par rapport à ceux du préscolaire/primaire. Au secondaire, l'enseignant est un spécialiste qui évolue dans un système morcelé dans lequel il lui est très difficile d'intégrer le français aux autres disciplines. Le programme étant chargé, il se concentre sur la lecture et l'écriture au détriment de 1'oral (Lafontaine, 2001, 2003; Lafontaine et Préfontaine, article soumis). L'oral est donc considéré comme un médium d'enseignement, ici au service de la lecture de contes, mais évalué comme s'il avait été enseigné. Tandis qu'au préscolaire/primaire, l'enseignant est le maitre d'œuvre des matières de base et peut ainsi créer des vases-communicants entre ces matières et la prise de parole. L'oral est donc plus facilement considéré comme un objet d'enseignement à ces ordres d'enseignement. Nous voyons encore une fois l'importance de considérer l'oral comme un objet d'enseignement à part entière (Dolz et Schneuwly, 1998; Lafontaine, 2001, 2003; Lafontaine et Préfontaine, article soumis) et d'intégrer l'oral à la vie de la classe (Nonnon, 1999; Le Cunff, 2004).

\subsection{Limites de la recherche}

Comme toute étude, notre recherche comporte des limites. Il est certain que l'éloignement géographique en est une importante. En effet, nous n'avons pas pu recueillir toutes les données en même temps. Malgré cela, comme une bonne partie de la collecte des données a eu lieu en présence des deux chercheures, nous avons pu nous entendre sur la façon de procéder afin d'éviter le maximum de biais dus à l'éloignement. Une autre limite peut être l'analyse des données du pays par la chercheure du pays. Toutefois, en croisant nos analyses entre nous et en les triangulant auprès des enseignants participants, nous avons $\mathrm{pu}$ valider nos interprétations. Nous rappelons qu'il était très important à nos yeux de respecter les différences culturelles de nos pays dans nos analyses et les exigences méthodologiques de nos institutions respectives afin, justement, de faire ressortir les éléments de comparaison entre la France et 
le Québec, ce que nous croyons avoir réussi. Dans la même veine, nous rappelons que le Québec ne dispose pas de structure pouvant libérer les enseignants de certaines périodes d'enseignement afin de participer à des recherches comme le fait la France. Il est certain que la validation des analyses faite par les enseignants français a été beaucoup plus approfondie que la validation de leurs homologues québécois.

\section{Conclusion}

Chez les enseignants interrogés et selon les observations de classe effectuées dans les deux pays, nous avons noté des distinctions importantes entre le préscolaire/primaire et le secondaire, et entre la France et le Québec. De plus, l'oral joue un rôle primordial dans la socialisation des élèves et permet de se construire (socialisation). La prise en charge la prise de parole se fait surtout de façon implicite (métacognition), et ce, particulièrement chez les élèves plus forts, car il n'est pas enseigné de façon systématique à tous les niveaux. Toutefois, l'oral est considéré comme un objet d'enseignement au préscolaire/primaire, puisqu'il est intégré aux activités quotidiennes de la classe. Cette façon de considérer l'oral se transforme au secondaire, où, dans notre étude, il semble plutôt utilisé comme un médium au service de la lecture des contes.

En outre, il s'avère primordial de travailler de concert avec les commissions scolaires au Québec, les conseillers pédagogiques et les enseignants québécois et français afin de faire modifier les pratiques trop morcelées au secondaire. Un travail serait aussi à considérer entre les chercheurs et les équipes ministérielles au sujet de l'organisation du temps à l'école secondaire afin de favoriser des vases-communicants entre les diverses matières faisant appel aux compétences langagières orales. Comme l'oral est considéré comme une compétence transversale au Québec, c'est dans la formation initiale des futurs enseignants et dans la formation continue des maîtres en poste que nous devons intervenir.

En somme, la coopération entre la France et le Québec dans notre recherche est un apport important à la didactique de l'oral qui, au-delà des frontières géographiques, a besoin d'être documentée scientifiquement par une étude comme celle-ci.

\section{Notes}

1. Le masculin est utilisé afin de garantir l'anonymat des participants.

2. Lafontaine, L. (2004). Rapport de recherche final. Projet de recherche francoquébécois Oral, savoirs et socialisation: Compétences langagières orales de la maternelle au collège, dans leur relation à la socialisation et aux apprentissages: prise de parole, conduites discursives, lexique, métacognition. Gatineau (Québec): Université du Québec en Outaouais.

Le Cunff, C. (2004), Oral savoirs socialisation, Rapport de recherche. Rennes (France) : Institut de formation universitaire de Bretagne. 
3. Les prénoms de tous les élèves ont été changés afin de respecter le contrat éthique décerné par le Comité éthique de la recherche de l'Université du Québec en Outaouais et d'assurer l'anonymat des participants.

\section{Références}

Amétooyona, R. (1995). L'enseignement du français au Bénin: à la recherche d'un modèle intégratif orienté vers l'enseignement de la compréhension et de la production de textes. Thèse de doctorat inédite, Université du Québec à Montréal.

Astolfi, J.-P. (1986). Les langages et l'élaboration de la pensée scientifique. Le Français Aujourd'hui, 74, 51-57.

Boisseau, P. et Raffestin, A. (1986). L'évaluation de l'expression orale. Rouen: Académie de Rouen.

Bronckart, J.-P. (1985). Le fonctionnement du discours. Un modèle psychologique et une méthode d'analyse. Paris : Éditions Delachaux et Niestlé.

Brossard, M. (1994). Quelques réflexions sur activités métalinguistiques et situations scolaires. Repères, 9, 29-36.

Charaudeau, P. (1992). Grammaire du sens et de l'expression. Paris: Hachette.

Culioli, A. (1973). Sur quelques contradictions linguistiques, Communications, 20, 83 91.

Dabène, M. (1987). L'adulte et l'écriture, contribution à une didactique de l'écrit en langue maternelle. Bruxelles : De Boeck-Wesmael.

Dolz, J. et Schneuwly, B. (1998). Pour un enseignement de l'oral. Paris : ESF.

Doutreloux, J.-M. (1985). Au programme: L'oral. Liaisons, 9(3), 29-37.

Doutreloux, J.-M. (1989). Prise en charge et évaluation de l'oral au primaire. Sherbrooke: Éditions du CRP.

Gadet, F. (1989). Le français ordinaire. Paris: Armand Colin.

Gadet, F. (1997). Il y a style et style. Le Français Aujourd'hui, 116, 23-31.

Halté, J.-F. (1992). La didactique du français, Coll. « Que sais-je? ». Paris : PUF.

Lafontaine, L. (2004). Rapport de recherche final. Projet de recherche franco-québécois Oral, savoirs et socialisation: Compétences langagières orales de la maternelle au collège, dans leur relation à la socialisation et aux apprentissages: prise de parole, conduites discursives, lexique, métacognition. Gatineau (Québec): Université du Québec en Outaouais.

Lafontaine, L. (2001). Élaboration d'un modèle didactique de la production orale en classe de français langue maternelle au secondaire. Thèse de doctorat inédite, Montréal, Université du Québec à Montréal.

Lafontaine, L. (2003). La culture scolaire en classe français langue maternelle au Québec: place de l'enseignement de l'oral et de la construction des connaissances, Actes du Colloque pluridisciplinaire international sur le langage et la construction des connaissances de l'IUFM de Bordeaux et de l'Université Victor-Segalen Bordeaux II, Bordeaux, France.

Lafontaine, L. et Préfontaine, C. (2004, article soumis). Modèle didactique de la production orale en classe de français langue maternelle au secondaire. Revue des sciences de l'éducation, 35 pages.

Lahire, B. (1993). Culture écrite et inégalités scolaires, sociologie de l'échec scolaire à l'école primaire. Lyon : PUL. 
Le Cunff, C. (2000). L'oral à l'école primaire et au collège : évolution dans le statut de l'oral et de la maîtrise de la langue. Dans A. Robert (Dir.). Les contenus d'enseignement en question : histoire et actualité. Rennes (France) : CRDP de Bretagne

Le Cunff, C. (2004, sous presse). Enseignement de l'oral, intégré aux apprentissages disciplinaires, et socialisation. $9^{\mathrm{e}}$ Colloque international de l'AIRDF, Le français : discipline singulière, plurielle ou transversale?

Le Cunff C. (2004), Oral savoirs socialisation. Rapport de recherche. Rennes (France) : IUFM de Bretagne.

Ministère de l'Éducation, Gouvernement du Québec. (1995). Programme d'études. Le français enseignement secondaire. Québec: Gouvernement du Québec.

Ministère de 1'Éducation, Gouvernement du Québec. (2001). Programme de formation de l'école québécoise. Éducation préscolaire et enseignement primaire. Québec: Gouvernement du Québec.

Ministère de 1'Éducation, Gouvemement du Québec. (2003). Programme de formation de l'école québécoise. Enseignement secondaire $1^{e r}$ cycle. Québec: Gouvernement du Québec.

Nonnon, É. (1999). L'enseignement de l'oral et les interactions verbales en classe: champs de référence et problématiques. Revue Française de Pédagogie, 129, 87131.

Ostiguy, L. et Gagné, G. (1992). La place de l'activité métalinguistique dans le développement de la langue orale. Québec Français, 84, 45-47.

Painchaud, G. (1992). «Littératie » et didactique de l'écrit en L2, Études de Linguistique Appliquée, 88, 55-66.

Pietro, J.-F. de et Wirthner, M. (1996). Oral et écrit dans les représentations des enseignants et dans les pratiques quotidiennes de la classe de français. Tranel, 25 , 29-49.

Rigault, A. (1971). Avant-Propos. Dans A. Rigault (Dir.). La grammaire du français parlé (pp. 5-12). Paris: Hachette.

Simard, C. (1997). Éléments de didactique du français langue première. Coll. «École en mouvement». Québec : Éditions du Renouveau Pédagogique.

Snow, C. E. (1983). Literacy and language: Relationships during the preschool years. Harvard Educational Review, 53(2), 165-189.

Tannen, D. (1982). Spoken and written language: Exploring orality and literacy. Norwood (N.J.): ABLEX Publishing Corporation.

Wirthner, M., Martin, D. \& Perrenoud, P. (1991). Parole étouffée, parole libérée. Neuchâtel (Suisse) : Delachaux et Niestlé. 


\section{Annexe: Contextes des activités filmées}

Lieu : Outaouais, Québec

Enseignant : $\quad$ EQ1

Niveau : Maternelle (5-6 ans)

Nombre d'élèves : 20

Matière : Toutes les compétences du programme préscolaire sont visées mais de façon plus particulière la compétence 4 : Communiquer en utilisant les ressources de la langue.

Activités d'oral : Causeries sur l'Halloween et sur les insectes

Matériel didactique utilisé: Chevalet et feuilles volantes, images, pictogrammes

Contexte de l'activité : L'enseignant fait réaliser deux causeries afin de développer une carte d'exploration sur les thèmes. Le but des activités est de favoriser l'émergence et l'expression de toutes les idées et connaissances sur les sujets afin de modifier ou de réaménager les aires de jeux de la classe selon le goût et les intérêts des élèves. Les élèves sont assis en demi-cercle devant l'enseignant, qui anime la causerie. Il gère les tours de parole et la discipline au sein du groupe. La causerie est une activité orale prévue au programme du préscolaire et du premier cycle du primaire.

\section{Objectifs des causeries :}

Communiquer en utilisant les ressources de la langue :

Produire un message (organiser ses idées, utiliser un vocabulaire approprié)

Comprendre un message (prêter attention au message, exprimer sa compréhension de l'information reçue)

Démontrer de l'intérêt pour la communication (engager la conversation et maintenir un contact avec son interlocuteur, respecter le sujet de conversation)

Agir avec efficacité dans différents contextes sur le plan sensoriel et moteur

Reconnaître des façons d'assurer son bien-être (prendre de bonnes postures)

Affirmer sa personnalité

Partager ses goûts, ses intérêts, ses sentiments et ses émotions

Interagir de façon harmonieuse avec les autres

Participer à la vie de groupe (exprimer ses idées, écouter les autres)

Construire sa compréhension du monde

Organiser l'information (exprimer ce qu'il connaît)

Mener à terme une activité

S'engager dans l'activité en faisant appel à ses ressources (manifester son intérêt, parler de ce qu'il connaît pour réaliser l'activité)

Lieu : Montérégie, Québec

Enseignant : EQ2

Niveau : $3^{\mathrm{e}}$ année du primaire

Nombre d'élèves : 26

Matière : Sciences et technologies

Activités d'oral : Expérience scientifique

Matériel didactique utilisé : manuel Les Fureteurs

Contexte de l'activité : Le midi précédant l'activité en groupe-classe, l'enseignant reçoit dans son local les six élèves experts. Ceux-ci ont été sélectionnés parmi les élèves qui désiraient faire vivre aux autres élèves de la classe, le lendemain, l'expérience scientifique. L'enseignant a préalablement préparé tout le matériel nécessaire à la réalisation de ladite activité. Les six élèves vivent donc l'expérience une première fois en 
compagnie de l'enseignant. Toutes les étapes sont réalisées selon la démarche scientifique : préparation, réalisation et intégration. Le soir venu, ces élèves apportent leur cahier à la maison (l'enseignant prend soin de diminuer la tâche déjà prévue en devoir pour ces élèves) afin de se préparer pour le lendemain. Le lendemain, chaque élève-expert se retrouve avec trois ou quatre élèves de la classe pour leur faire vivre l'activité. L'expert fait tout. Il prépare son matériel, organise, donne la parole, aide et soutient ses apprentis. Son rôle est assez complexe. Il doit laisser de la place aux autres sans perdre de vue sa tâche de meneur. Il prépare son matériel, organise, donne la parole, aide et soutient ses apprentis.

Pour l'enseignant, c'est un moment idéal pour circuler, observer, prendre des notes sur la prise de parole de ses élèves, autant experts qu'apprentis. Aussi, cela lui permet d'avoir un regard sur le travail d'équipe. D'après ses observations, il pourra faire un retour sur un élément relatif à la prise de parole lorsqu'il se retrouvera avec son groupe à la fin de l'activité.

Le retour en grand groupe se fait en deux temps : on s'attarde premièrement aux compétences à l'oral. On discute du climat dans les équipes, de la prise de parole. On se donne des moyens pour améliorer l'esprit d'équipe ou pour rendre la prise de parole plus équilibrée entre les membres de l'équipe et ainsi rendre plus agréable et plus efficace la réalisation de l'activité. Dans un deuxième temps, les connaissances et les techniques utilisées sont discutées.

L'expérience scientifique n'est pas une activité d'oral préconisée par le programme d'études de deuxième cycle du primaire. Toutefois, à travers cette expérience, l'enseignant fait travailler une autre activité au programme : la discussion.

\section{Objectifs:}

Faire preuve de leadership (animateur)

Apprendre à écouter l'autre

Apprendre à respecter son tour de parole

Apprendre à donner des consignes

Apprendre à respecter l'opinion de l'autre

Apprendre à relancer la discussion

Apprendre à interagir pendant la discussion (questions, etc.)

\section{Lieu : Outaouais, Québec}

Enseignant : $\quad \mathrm{EQ} 3$

Niveau : $5^{\mathrm{e}}$ année (10-11 ans)

Nombre d'élèves : 23

Matière : Français langue d'enseignement

Activités d'oral : Discussions de groupe sur la fête de Noël ainsi que sur le bilan de l'année scolaire et les vacances d'été

Matériel didactique utilisé: Un animal en peluche que les élèves s'échangent afin de respecter les tours de parole. Le locuteur l'a en main et le passe au prochain locuteur.

Contexte de l'activité : Les élèves sont assis en demi-cercle. L'enseignant anime la discussion et donne les tours de parole. Afin de s'assurer de l'écoute des élèves, il remet un animal en peluche à celui qui parle. Lorsqu'il y a changement de tour de parole, l'élève doit donner l'animal au nouveau locuteur.

$\mathrm{Au}$ début, les élèves sont intimidés par la caméra et l'enseignant doit régulièrement susciter leur participation. Plus la discussion avance, plus les élèves prennent naturellement la parole et s'intéressent aux propos des locuteurs. primaire.

La discussion est une activité orale prévue au programme du troisième cycle du 


\section{Objectifs:}

Apprendre à écouter l'autre

Apprendre à respecter son tour de parole (animal en peluche)

Apprendre à donner son opinion

Apprendre à respecter l'opinion de l'autre

Apprendre à relancer la discussion

Apprendre à interagir pendant la discussion (questions, etc.)

\section{Lieu : Montérégie, Québec}

\section{Enseignant : $\quad$ EQ4}

Niveau : $1^{\text {re }}$ secondaire $(12-13$ ans)

Nombre d'élèves : 8

Matière : Français langue d'enseignement

Activité : Discussions sur des contes d'Andersen

Matériel didactique utilisé: Contes, grille d'autoévaluation du locuteur, grille d'autoévaluation de l'observateur, feuilles de notes de lecture, consignes pour un oral, liste de comportements possibles lors de la discussion

\section{Contexte de l'activité :}

Les élèves réalisent les deux discussions sommatives de l'année scolaire. Les élèves sont divisés en groupes de discussion de quatre personnes et sont jumelés à quatre observateurs. La discussion terminée, les rôles sont inversés. Chaque observateur évalue un élève et lui donne une rétroaction écrite ou orale de ses forces et de ses faiblesses. L'enseignant évalue chacun des participants à la discussion et prend en considération la note de l'observateur.

Afin de se préparer à discuter, les élèves ont eu deux semaines pour lire un recueil de contes d'Andersen. Ils devaient, en devoir, prendre des notes de lecture sur des éléments suscitant la discussion : personnages, morale, vocabulaire, magie, etc. Lors de la discussion, ils devaient comparer leurs réponses au sujet d'un seul conte, choisi au hasard par l'enseignant, et arriver à un consensus pour chacune des questions.

La discussion d'œuvres littéraires est un activité langagière orale au programme de la première secondaire.

\section{Objectifs:}

Apprendre à écouter l'autre

Apprendre à attendre son tour de parole

Apprendre à donner son opinion

Apprendre à respecter l'opinion de l'autre

Apprendre à socialiser en groupe

Apprendre à apprécier des œuvres littéraires

Apprendre à arriver à un consensus

\section{Lieu : Normandie, France}

\section{Enseignant : $\quad$ EF1}

Niveau : Matemelle Petite Section ( $2-3 a n s)$

Nombre d'élèves : 21

Matière : Français langue d'enseignement

Activités : Corpus 1 : Météo puis fiche sur la poule et récit d'un élève; Corpus 2 : Discussion de groupe sur le jardin public

Matériel didactique utilisé: Corpus 1 : Météo : Deux élèves sortent pour donner des informations dans un ordre établi qui sont notées par un troisième élève à l'aide d'un matériel spécifique qui se fixe sur un tableau. Fiche: Les élèves apportent les 
informations qu'ils ont sur l'animal qui fait l'objet du travail. Un élève apporte une information que l'enseignant invite à développer en récit.

Corpus 2 : Discussion sur le jardin public : les savoirs ou hypothèses sont notées sur un tableau blanc par l'enseignant.

\section{Contexte de l'activité :}

Ces activités d'oral avec tous les élèves se déroulent chaque jour (rituel). Un élève ou deux selon l'activité ont à tour de rôle une place « vedette ». L'activité de discussion dans le cadre de la découverte du monde évolue à la seconde période (corpus 2) mais concerne toujours la mobilisation des savoirs sur un objet du monde : le jardin public où le groupe classe doit se rendre pour faire des observations.

\section{Objectifs de l'activité :}

Parler en attendant son tour

Retenir ce que je veux dire pour différer

\section{Lieu : Grande banlieue de Paris, France \\ Enseignant : $\quad$ EF2}

Niveau : Maternelle (5 - 6 ans)

Nombre d'élèves : 20

Matière : Français langue d'enseignement

Activités d'oral : Vivre ensemble (apprentissage de la citoyenneté), découverte du monde, langage

Matériel didactique utilisé: Les objets

Contexte de I'activité : Un questionnement métalinguistique de l'enseignant permet de construire le cadrage et contribue à faire de la séquence une séquence d'apprentissage des conduites langagières et linguistiques. Les interventions de cette nature se situent au début mais surtout à la fin, comme retour sur les productions, à réinvestir dans les séquences suivantes.

Les élèves prennent le relais par la régulation notamment sur le bruit, le volume de voix et les redites interdites. Les élèves ont des modalités et des compétences d'intervention différentes. Cette différence est prise en compte dans l'étayage effectué par l'enseignant.

Des prises en compte de la parole de l'autre, des réactions, (certaines empreintes de civilité d'autres non) pour le respect des règles sont l'amorce d'une conduite de discussion.

Les règles générales, l'écoute, le respect de formes linguistiques, le retrait progressif de l'enseignant, la réflexion métalinguistique contribuent à construire des compétences pour la discussion. L'enseignant intervient peu sauf sur la construction métalangagière et au départ sur la régulation.

\section{Objectifs de l'activité :}

Mettre en vedette un élève sur une initiative de sa part (il a donc un rôle "vedette »: répondre aux questions ou présenter l'objet, dans une situation ritualisée qui doit lui donner les repères pour sa conduite discursive)

Faire entrer les élèves dans une conduite définie par des règles particulières et générales.

Pour la devinette : poser des questions efficaces dans les formes (phrase) à l'interlocuteur choisi pour le rôle vedette

Répondre par une phrase

Pour la présentation de l'objet : poser des questions avec des phrases à l'interlocuteur défini par la situation 
Lieu : Normandie, France

Enseignant : $\quad$ EF5

Niveau : Primaire cycle 3 ( $8-10$ ans)

Nombre d'élèves : 24

Matière : Français langue d'enseignement

Activités : Débat (apprentissage de la citoyenneté)

Matériel didactique utilisé: Documents de presse, enregistrement vidéo pour la critique

Contexte de l'activité : Pour définir la fonction d'un débat, les élèves font le choix du sujet. Ils définissent ensuite le rôle du Président et du Secrétaire et font le débat, qui est filmé. L'enseignant ne dit rien. Après, le maître et les élèves font des réflexions immédiates sur le débat et une réflexion différée sur le débat en visionnant une partie de la vidéocassette.

\section{Objectifs de l'activité :}

Oser parler/parole libérée

Écouter les autres

Argumenter

Développer, affiner sa pensée

Questionner

Rester dans le sujet

Proposer des solutions au problème posé

Rester logique dans ses propositions

S'appuyer, enchaîner sur les propos de l'autre (pour acquiescer, pour démentir immédiatement ou en différé)

Respecter toutes les idées

Être capable de porter des jugements sur des actes. 